\title{
Comparación del tiempo de cepillado de la técnica habitual con la técnica de Bass en estudiantes de Odontología
}

\author{
Nohelia María Pérez-Bejarano, Marta Inés Ferreira-Gaona, Viviana Soledad Alarcón-González, Clarisse \\ Virginia Díaz-Reissner, Maia Macarena Arce-Mena, Natalia Cristina Cueto-González, David Alessandro \\ Urquhart-Barreto, Luis Enrique Ledesma-Amarilla
}

Facultad de Odontología. Universidad del Pacífico. Paraguay

Cómo referenciar este artículo/ How to reference this article: Pérez-Bejarano NM, Ferreira-Gaona MI, Alarcón-
González VS, Díaz-Reissner CV, Arce-Mena MM,
Cueto-González NC, et al. Comparación del tiempo de
cepillado de la técnica habitual con la técnica de Bass en
estudiantes de Odontología. Mem. Inst. Investig. Cienc.
Salud. 2016;14(3):81-85

\section{RE S U M E N}

El proceso de cambio de hábitos comienza con la educación del paciente, promocionando el desarrollo de un aceptable control de la placa. Dentro de este contexto, varios métodos se emplean para ejecutarlo, sin embargo el valor de cepillado en la prevención depende no solo del tipo de cepillo de dientes, sino también en la técnica de cepillado apropiada y del tiempo que se dedique a la ejecución del mismo. El objetivo del estudio fue comparar los tiempos de cepillado con técnica habitual y de Bass en estudiantes de la Cátedra de Odontología Preventiva de la Universidad del Pacífico. El estudio es cuasi-experimental e incluyó la participación de 21 estudiantes del primer año de la carrera de Odontología matriculados en el 2014.En la clase previa al desarrollo del contenido programático "Control mecánico de placa microbiana", se invitó a participar del estudio a todos los estudiantes. Con un espejo de rostro y el cepillo personal del estudiante se le solicitó a cada uno que proceda a cepillarse con su técnica habitual y posterior a la clase, con la de Bass, se registraron en la ficha los tiempos que cada uno tomo, en segundos, a través cronómetro digital. El tiempo promedio con la técnica de Bass fue significativamente $(p<0,0001)$ mayor

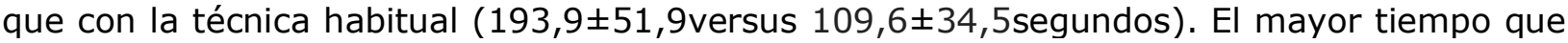
se ocupa para el cepillado con la técnica de Bass puede resultar particularmente importante, considerando que el ritmo actual de vida del estudiante y de la población en general implica una carrera contra reloj, limitando así las posibilidades de un adecuado control mecánico de la placa microbiana, reconocida como uno de los más importantes factores cariogénico y periodontopático.

Palabras clave: Cepillado Dental/métodos, Estudios de Tiempo y Movimiento, Placa dentaria

\section{Comparison of brushing time with the usual technique and Bass technique on dental students}

\section{A B S T R A C T}

The process of changing habits starts with patient's education, promoting the development of an acceptable plaque control. Within this context, several methods are used, however, the value of brushing in prevention depends not only on the type of toothbrush, but also in the art of proper brushing and time devoted to the execution of it. The aim of this study was to compare regular brushing times and Bass techniques in students of the Department of Preventive Dentistry at the Universidad del Pacífico. The Quasi-experimental study involves the participation of 21 first year students of the career of Dentistry enrolled in 2014. In the previous class to the development of the content "Mechanical control of microbial plaque", all students were invited to participate in this study. Using a mirror and personal thoot brushe very student was asked to proceed to Fecha de recepción: setiembre 2016. Fecha de aceptación: noviembre 2016 Autor correspondiente: Clarisse Virginia Díaz Reissner. Facultad de Odontología Universidad del Pacífico. Asunción, Paraguay 
brushing with conventional technique and after class using Bass technique, time spent in every process was measured by a digital stopwatch and written in the file. The average time using Bass technique was significantly greater than $(p<0.0001)$ using the standard technique (193.9 \pm 51.9 versus $109.6 \pm 34.5$ seconds). More time dealing for brushing using Bass technique may be particularly important given the current pace of student life and the population generally involves a time rush, limiting the possibilities of a suitable mechanical control of microbial plaque, recognized as one of the more important cariogenic and periodontopathic facts.

Keyword: Tooth brushing/methods - Time and Motion Studies - Dental Plaque

\section{INTRODUCCIÓN}

Actualmente, las enfermedades bucales prevalentes siguen siendo la caries y las patologías periodontales; las mismas tienen una etiología multifactorial(1-3). Sin embargo, el papel de la placa microbiana o placa dental, se torna fundamental e indispensable para que las mencionadas enfermedades se inicien y progresen(4), por lo que se debe considerar que su control eficaz puede mantener el equilibrio de la microbiota oral y evitar o minimizar los daños sobre las estructuras dentales y/o periodontales(5).Además, resulta importante destacar que pese a la cantidad de medidas preventivas para el control de la placa dental existentes, que pueden llevarse a cabo a través de medios mecánicos, muchas personas aún presentan dificultades para realizarlo correctamente, inherentes a un factor biológico o de falta de motivación(6).Por lo expuesto, las patologías bucales siguen ejerciendo una tremenda carga social, económica y financiera en los sistemas de salud a escala global(7).

Son diversos los métodos que pueden emplearse para el control de placa, y en lo que respecta a la eficacia del cepillado, no sólo tiene que ver con el tipo de cepillo de dientes, sino también más bien con la técnica de cepillado apropiada (8), la sistematización de la misma y tiempo que se dedique a la ejecución dela misma(6).Pues no se trata simplemente de frotar los dientes en el intento de limpiarlos, sino más bien importa la técnica aplicada, la duración del proceso, su organización y factores físicos tales como la fuerza que afectan la eficiencia del control de placa(6,9). Estudios sobre la efectividad de las diferentes técnicas han demostrado que las más utilizadas son la de Ramfjörd, Stillman modificado y la técnica de Bass; sin demostrar clara ventaja para cualquiera de los métodos en cuanto a efectividad. Sin embargo, es probable que la técnica de "lavado" o personal, sea el método de cepillado más simple y común(10). Cabe mencionar que la eficiencia en el control de la placa dental, se complementa con elementos de uso interdental y pastas dentales fluoradas.

Se debe considerar que el proceso de cambio de hábitos comienza con la educación al paciente, promocionando el desarrollo de un control de la placa adaptado a sus habilidades y marcando los momentos adecuados, siempre haciendo hincapié en los cambios positivos en el comportamiento(10).La enseñanza del control mecánico de la placa es de suma importancia; de las múltiples opciones ya mencionadas, la técnica de cepillado de Bass viene siendo una de las más promovidas por su simpleza y efectividad, tal como lo demostró Schmidt et al.(11), concluyendo que la explicación de la técnica de cepillado resulta ser sencilla, práctica y efectiva para el control de la placa dental.

La herramienta básica de este proceso es la posición del cepillo de dientes manual convencional cuyo diseño es atribuido al Dr. Robert Hutson (periodoncista californiano), quien en la década de 1950 desarrolló el "flattrimmed", diseñado para limpiar todas las partes de la cavidad oral. Los cepillos de dientes convencionales planos recortados basados en el diseño original tienen una buena capacidad de control de placa cuando su uso es adecuado. Posteriormente, limitaciones en cuanto a técnicas y tiempo de cepillado hicieron necesarios cambios en el patrón de cerdas para mejorar el rendimiento, especialmente en superficies proximales y a lo largo del límite gingival(12).Estudios han demostrado que en todas las situaciones en las que el control de la placa fue supervisado y reforzado con programas a largo plazo resultaron efectivos, mientras que en instrucciones de una sola sesión, los resultados no fueron favorables $(6,10)$.

En base a lo expuesto, el presente estudio fue diseñado con el objetivo de comparar los tiempos destinados al cepillado usando una técnicas de higiene habitual y de Bass, ambas supervisadas por los investigadores, en estudiantes de la Cátedra de Odontología Preventiva de la Universidad del Pacífico 


\section{MATERIALES Y MEÉTODOS}

Formaron parte del estudio estudiantes de Odontología de $1^{\text {er }}$ año matriculados en la Universidad del Pacífico en el año 2014. Participaron los mayores de 18 años presentes en una única jornada asignada para la recolección de datos; incluyéndose sólo los que contaban con sus elementos de higiene personal. El protocolo fue aprobado por el Comité de Ética en la Investigación de la Universidad del Pacífico.

En la clase previa al desarrollo del contenido programático "Control mecánico de placa microbiana", se invitó a participar a todos los estudiantes. Se recurrió a un muestreo de casos consecutivos. Una vez obtenido el consentimiento, con un espejo de rostro y el cepillo personal del estudiante se le solicitó a cada uno que proceda a cepillarse con la técnica habitualmente utilizada y se registró en la ficha el tiempo ocupado en segundos medido a través de un cronómetro digital: desde la introducción del cepillo a la boca hasta que el estudiante indicó la finalización del procedimiento.

Posteriormente, se desarrolló la clase con las instrucciones específicas de la técnica de Bass y se solicitó individualmente la aplicación de la misma para el control de la correcta secuencia, mostrando y corrigiendo cualquier error en esa única oportunidad. Finalmente, se registró la aplicación de la técnica completa sin pausas, con el mismo cronómetro, desde el momento en que se le indicó iniciar hasta la conclusión del procedimiento.

Para asegurar el registro, un solo examinador (docente a cargo del desarrollo del contenido) verificó que la secuencia de la técnica sea la apropiada y se hizo cargo del uso del cronómetro de registro. Se aseguró que todos los registros se efectuaran con el mismo aparato digital.

Las variables consideradas fueron el tiempo de cepillado por técnica habitual y con técnica de Bass y se registró el tiempo en segundos.

La carga de los datos se hizo en una base de datos validada, diseñada en el programa Excel de Microsoft ${ }^{\circledR} 2011$ de Microsoft Corporation. Se aplicó estadística descriptiva para resumir las variables de interés: medidas de tendencia central y de dispersión. La estadística analítica consistió en la prueba T de Student, utilizando el software EpiInfo ${ }^{\mathrm{TM}}$ 7, desarrollado por el Centers for Desease Control and Prevention (CDC)(13) y el gráfico con IBM SPSS 22 versión de prueba. Se consideró significativa una $p<0,05$.

\section{RESULTADOS}

Participaron del estudio 21 estudiantes que cumplieron con los criterios establecidos. El tiempo promedio para el cepillado en el modo habitual fue de $109,6 \pm 34,5$ segundos. El mínimo de tiempo ocupado para el efecto fue de 60 segundos y el más prolongado fue de

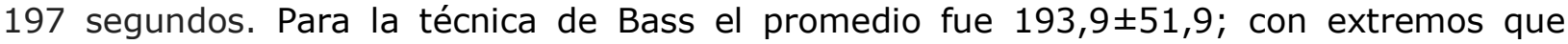
oscilaron entre 120 y 292 segundos. La diferencia de promedios fue de 84,3 segundos entre una y otra técnica, siendo esta estadísticamente significativa $(t=6,38020 ; p<0,0001)$ (Figura 1).

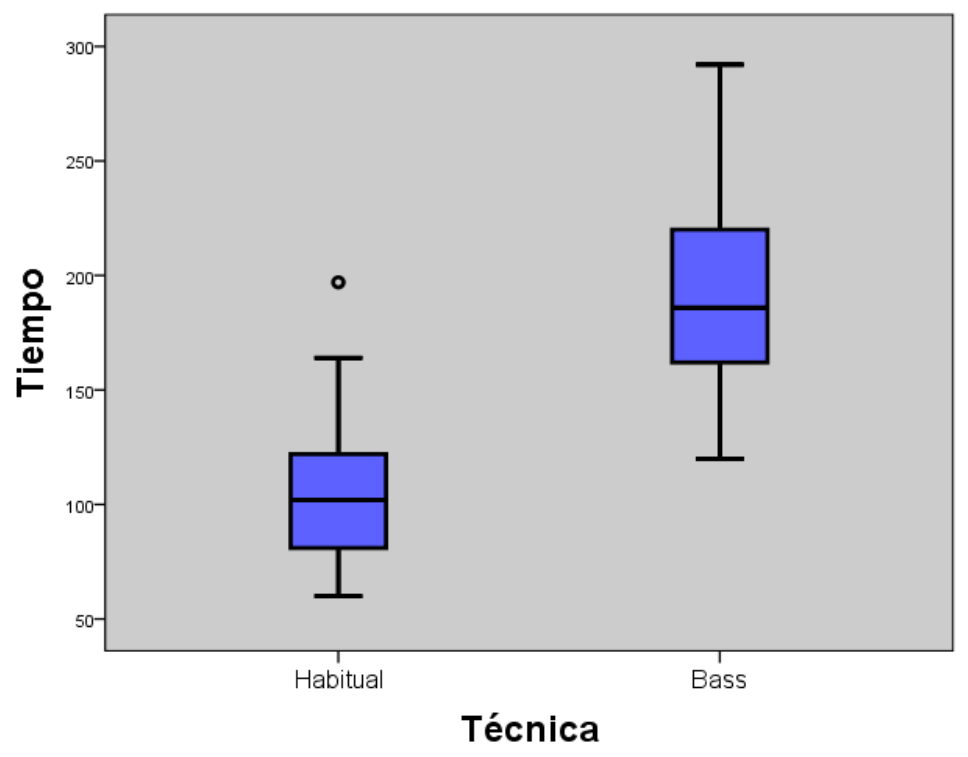

Figura 1. Tiempos de cepillado por técnica habitual y técnica de Bass. Estudiantes de Odontología del 1er año. Universidad del Pacífico Privada. Paraguay-2014 


\section{DISCUSIón}

El control mecánico de placa microbiana representa una de las medidas más efectivas en la prevención de la caries dental y las periodontopatías, y en tal sentido exige un tiempo para efectuarlo de manera adecuada. Recomendaciones profesionales para la higiene oral individual mencionan una frecuencia de al menos dos veces por día por espacio de 2 a3 minutos con fuerza suave con la técnica de Bass o modificaciones de ella $(1,3,14,15)$.

En el presente estudio se buscó identificar si existe diferencia entre los tiempos promedios dedicados al cepillado dental, cuando se ocupa con una técnica habitual y otra que demande la aplicación de una técnica, en este caso la de Bass, ambas, supervisadas por un experto.

El promedio determinado en el uso de la técnica habitual, previo a la demostración teórico

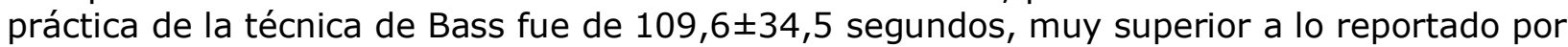
Gansset al(6) sobre 103 adultos sin instrucción previa (96,6 $\pm 36,0$ segundos), considerando que la población evaluada en el mencionado estudio, excluía a los que tenían instrucción en cualquier área de la salud, en tanto esta muestra incluyó a estudiantes de los primeros cursos de Odontología; quienes si bien aún no habían sido instruidos en la importancia del tiempo de cepillado, ya poseían algunos previos conceptos pero no así incorporada la destreza para la técnica de cepillado efectuada.

Se observaron tiempos particularmente extensos en algunos de los participantes, lo que pudiera deberse a la conciencia de ser observados, fenómeno que fue reportado por Macgregor et al.(16) en un estudio en 90 adultos en el que se observó que los sujetos que se sabían observados, prolongaron significativamente el tiempo de cepillado. Rugg-Gunn \& Macgregor(17) obviaron este fenómeno en otro estudio en 88 sujetos obteniendo tiempos más reducidos.

Schiff et al.(18)en un estudio longitudinal en 144 sujetos demostraron que 60 segundos dedicados al cepillado puede reducir (con o sin uso de hilodental) significativamente la presencia de placamicrobiana, mejorando la situación gingival, de allí la importancia de incorporar en el concepto de higiene el tiempo que se debe asignar al procedimiento.

Se concluye que el tiempo que ocupa el cepillado con técnica de Bass es significativamente mayor que con la técnica habitual; esto puede resultar particularmente importante, considerando que el ritmo actual de vida del estudiante y de la población en general implica una carrera contra reloj, limitando así las posibilidades de un adecuado control mecánico de la placa microbiana.

Considerando que el control de la placa representa uno de los principales pilares de la prevención de las patologías bucales prevalentes, el tiempo que se debe dedicar a efectuarlo correctamente es de suma importancia.En este sentido se debe considerar un especial énfasis en la motivación del ajuste de tiempo en el proceso de enseñanza de la técnica de cepillado de Bass y considerar la sistematización de cualquier técnica.

\section{REFERENCIAS BIBLIOGRAFICAS}

1. Baca García P. Caries: Fundamentos para su prevención y control. En: Cuenca Sala E, Baca García P, editores. Odontología preventiva comunitaria: principios, métodos y aplicaciones. 3ra ed. Barcelona: Elsevier Masson; 2005. p. 19-40.

2. Lindhe J. Periodontología Clínica e Implantología Odontológica. 4ta ed. Buenos Aires: Médica Panamericana; 2008.

3. Collet AM, Guglielmotti MB. Patologías dentarias de etiología no infecciosa. En: Barrancos Money J, Barrancos Money P, editores. Operatoria dental Integración clínica. Buenos Aires: Médica Panamericana; 2009. p. 291-5.

4. Marcatoni M. Caries dental. Antimicrobianos y vacunas para su control. En: Negroni B, editor. Microbiología Estomatológica Fundamentos y guía para su práctica. 2da ed. Buenos Aires: Médica Panamericana; 2010. p. 247-3.
5. Rioboo R. Higiene y prevención en odontología: individual y comunitaria. Madrid: Avances Médico-Dentales; 1994.

6. Ganss C, Schlueter N, Preiss S, Klimek J. Tooth brushing habits in uninstructed adults-frequency, technique, duration and force. Clin Oral Investig. 2009;13(2):203-8. Doi: $10.1007 /$ s00784-008-0230-8

7. OMS|Salud Bucodental [Internet]. WHO. 2007 [citado 2 de octubre de 2013]. Recuperado a partir de: http://www.who.int/mediacentre/factsheets /fs318/es/index.html

8. Damle SG, Patil A, Jain S, Damle D, Chopal N. Effectiveness of supervised toothbrushing and oral health education in improving oral hygiene status and practices of urban and rural school children: A comparative study. J Int Soc Prev Community Dent. 2014;4(3):175-81. Doi: 10.4103/2231-0762.142021.

9. Dávila L, Sosa L, Ramírez D, Arteaga S, Palacios M. Evaluación de la eficacia de la 
Pérez-Bejarano et al Comparación del tiempo de cepillado de la técnica habitual con la Bass técnica de cepillado de bass modificada a través de un metodo de enseñanza intraoral y otro extraoral. Rev Odontológica Los Andes. 2012;7(2):21-33.

10. Nassar PO, Bombardelli CG, Walker CS, Neves KV, Tonet K, Nishi RN, et al. Periodontal evaluation of different toothbrushing techniques in patients with fixed orthodontic appliances. Dent Press J Orthod. 2013;18(1):76-80. Doi: http://dx.doi.org/10.1590/S217694512013000100017

11. Schmidt JC, Bux M, Fillipuzzi-Jenny E, Kulix EM, Waltimo T, Weiger Ret al. Influence of time, toothpaste and saliva in the retention of Streptococcus mutans and Streptococcus sanguinis on different toothbrushes. J Appl Oral Sci. 2014;22(3):152-8.

12. Cugini, $M$, Warren, PR. El Oral-B CrossAction manual Cepillo de dientes: A 5 años de revisión de la literatura. J Can Dent Assoc. mayo de 2006;72(4):323a - 323k.

13. Center of Disease Control Prevention. Epi Info TM [Internet]. [citado 4 de mayo de 2015]. Recuperado a partir de: http://wwwn.cdc.gov/epiinfo/7/

14. Zeif T, BóvedaC. Cariología: prevención, diagnóstico y tratamiento contemporáneo de la caries dental. Caracas: Actualidades Médico Odontológicas Latinoamérica; 1997.

15. Fellman TG, Debates TE, Samuel W, Bergan CR. ¿Cuánto tiempo debo cepillarme los dientes? [Internet]. South University Dental Associates. 2015 [citado 19 de mayo de 2015]. Recuperado a partir de: http://southuniversitydental.com/blog/howlong-should-i-brush-my-teeth

16. Macgregor IDM, Rugg-Gunn AJ. Effect of filming on tooth brushing performance in uninstructed adults in north-east England. Community Dent Oral Epidemiol [Internet]. 1986;14(6):320-2. Doi: 10.1111/j.16000528.1986.tb01082.x

17. Rugg-Gunn AJ, Macgregor IDM. A survey of toothbrushing behaviour in children and young adults. J Periodontal Res. 1978;13(4):382-9.

18. Schiff T, Proskin HM, Zhang YP, Petrone M, DeVizio W. A clinical investigation of the efficacy of three different treatment regimens for the control of plaque and gingivitis. J Clin Dent. 2006;17(5):138-44. 